\title{
Improvements in Shoulder Research and Rehabilitation
}

\author{
Kellie C. Huxel Bliven and Kelsey J. Picha
}

It has been well over a decade since the Journal of Sport Rehabilitation published a shoulder thematic issue; thus, we are excited to highlight current research of colleagues contributing rehabilitation-focused evidence in this area. Our goal is that the compilation of articles presented here will aid researchers and clinicians in continuing the pursuit of improving care and rehabilitation for patients with shoulder conditions.

In preparing this thematic issue, titled "Current Trends in Shoulder Rehabilitation," and reflecting on the current state of shoulder rehabilitation research, several themes became apparent that we want to draw attention to as you read the articles. While studying muscle activation and strength has been persistent over time, the nature in which it is being studied has evolved. No longer are muscles being examined in isolation; rather primary and secondary movers, force couples, and compensatory muscles are considered simultaneously and collectively. This shift toward a more comprehensive approach, in both muscle activation and strength testing, provides a more detailed and functional understanding of the shoulder. It is well established that supraspinatus muscle alterations exist in the presence of shoulder pain and pathology; however, recent focus on the infraspinatus muscledeficiencies, alterations and adaptations, and response to treatment -is reflected in 4 of this issue's articles (Contemori and Biscarini, ${ }^{1}$ Kamali et al, ${ }^{2}$ Pexa et $\mathrm{al}^{3}{ }^{3}$ and $\mathrm{Yu}$ et $\mathrm{al}^{4}$ ). We frequently ask clinical questions regarding position and technique for optimal strengthening and articles in this issue continue the pursuit. Abe et $\mathrm{al}^{5}$ provide guidance on strength testing with dynamometers; Yu et $\mathrm{al}^{4}$ examine positioning relative to infraspinatus torque and activation; and Kim et $\mathrm{al}^{6}$ present strength and muscle activation differences in individuals with versus without a winged scapula, a common impairment in patients with shoulder pain and injury.

We also continue to strive for and ask relevant questions about the effectiveness of therapeutic interventions. While many researchers have sought to learn more about how interventions, such as taping and myofascial trigger point release may be used to decrease pain, target muscle activity and increase motion to promote and enhance short- and long-term therapeutic outcomes, the evidence continues to be inconclusive. Gulpinar et $\mathrm{al}^{7}$ and Leong and $\mathrm{Fu}^{8}$ present their research on the effects of rigid and kinesio taping in overhead athletes and rigid scapular taping in athletes with rotator cuff tendinopathy, respectively, suggesting tape may be advantageous in short-term improvements in shoulder motion, including glenohumeral internal rotation and subacromial space. Dry needling's emergence as an intervention continues to gain attention, and Kamali et $\mathrm{al}^{2}$ shed insight about the effect of its

Huxel Bliven is an Associate Editor of the Journal of Sport Rehabilitation (JSR); and Picha is a JSR Editorial Board member. Both are with A. T. Still University. Huxel Bliven (kbliven@atsu.edu) is corresponding author. location relative to a myofascial trigger point on pain and disability in overhead athletes with shoulder impingement.

Excitement surrounds the articles focusing on specific patient populations within this shoulder thematic issue. Ability to rehabilitate the pathologic shoulder is dependent on understanding the unique presentation of outcomes under functional conditions and relevant interventions. Given the high prevalence of injury in overhead athletes, studies by Contemori and Biscarini, ${ }^{1}$ Kamali et $\mathrm{al}^{2}{ }^{2}$ and Guney Deniz et $\mathrm{al}^{9}$ examining the impact of overhead athletes with secondary suprascapular nerve neuropathy, subacromial impingement, and factors that impact acromiohumeral distance, respectively, provide insight about compensatory strategies and specific recommendations for treatment approaches in patients. Murphy et al $^{10}$ detail a clinically-focused case report of a successful accelerated return-to-play protocol following Latarjet shoulder reconstruction. Furthermore, this issue continues to make efforts toward greater awareness about injuries and rehabilitation of athletes with physical disabilities. Fairbairn and Huxel Bliven's ${ }^{11}$ critically appraised topic provides evidence on how sport impacts injury incidence in elite wheelchair athletes. In a systematic review, Melo et al ${ }^{12}$ provide best evidence on physical training and upperlimb strength in individuals with paraplegia. Continued research on paraplegic and wheelchair athlete populations is needed to better inform clinicians about unique patient needs.

Finally, the most important apparent theme in this issue is the overall progress toward improved research designs and measured outcomes. Not only do the studies published in this issue address relevant research questions, but also they answered their hypotheses using well-controlled study designs. Both the systematic review by Melo et al $^{12}$ and the critically appraised topic by Fairbairn and Huxel Bliven ${ }^{11}$ synthesize available evidence and make best practice recommendations from the body of literature. Randomized controlled or clinical trials were used by Gulpinar et al, ${ }^{7}$ Kamali et $\mathrm{al}^{,}{ }^{2}$ and Leong et $\mathrm{al},{ }^{8}$ whereas cross-sectional designs with randomization, when appropriate, were used by the remaining authors. The elevation of study designs toward higher levels of evidence, as noted by the systematic review and randomized trials, is noteworthy and displays significant advancements in the quality of shoulder rehabilitation research. In addition, the relevance of the studies presented in this issue is apparent by the integration of laboratory, clinical, and patient-reported outcome measures used to answer the research questions.

As you read the articles in this special issue, not only do we hope you learn from each study individually, but are also able to appreciate the overall themes of muscle activation and strength, effectiveness of therapeutic interventions, patient populations, and improved research designs and measured outcomes. Each of these themes contributes to the goal of continued pursuit toward improved care and rehabilitation for patients with shoulder pathology. We hope you may learn from and integrate findings from the articles in this shoulder thematic issue to enhance your research and practice. 


\section{References}

1. Contemori S, Biscarini A. Isolated infraspinatus atrophy secondary to suprascapular nerve neuropathy results in altered shoulder muscles activity. J Sport Rehabil. 2019;28:219-228. doi:10.1123/ jsr.2017-0232

2. Kamali F, Sinaei E, Morovati M. Comparison of upper trapezius and infraspinatus myofascial trigger point therapy by dry needling in overhead athletes with unilateral shoulder impingement syndrome. J Sport Rehabil. 2019;28:243-249. doi:10.1123/jsr.2017-0207

3. Pexa BS, Ryan ED, Hibberd EE, Teel E, Rucinski TJ, Myers JB. Infraspinatus cross-sectional area and shoulder range of motion change following live-game baseball pitching. J Sport Rehabil. 2019;28:236-242. doi:10.1123/jsr.2017-0158

4. Yu I-Y, Lee D-K, Kang MJ, Oh J-S. Effects of 3 infraspinatus muscle strengthening exercises on isokinetic peak torque and muscle activity. J Sport Rehabil. 2019;28:229-235. doi:10.1123/jsr.2017-0110

5. Abe T, Loenneke JP, Thiebaud RS, Loftin M. The bigger the hand, the bigger the difference? Implications for testing strength with 2 popular handgrip dynamometers. J Sport Rehabil. 2019;28:278-282. doi:10.1123/jsr.2017-0189

6. Kim J-S, Kim M-H, Ahn D-H, Oh J-S. Comparison of shoulder protraction strength and electromyography activity of serratus anterior and pectoralis major in subjects with or without a winged scapula. J Sport Rehabil. 2019;28:272-277. doi:10.1123/jsr.20180059

7. Gulpinar D, Ozer ST, Yesilyapra SS. Effects of rigid and kinesio taping on shoulder rotation motions, posterior shoulder tightness, and posture in overhead athletes: a randomized controlled trial. J Sport Rehabil. 2019;28:256-265. doi:10.1123/jsr.2017-0047

8. Leong HT, Fu SN. The effects of rigid scapular taping on the subacromial space in athletes with and without rotator cuff tendinopathy: a randomized controlled study. J Sport Rehabil. 2019;28:250255. doi:10.1123/jsr.2017-0287

9. Guney-Deniz H, Harput G, Toprak U, Duzgun I. Relationship between middle trapezius muscle activation and acromiohumeral distance change during shoulder elevation with scapular retraction. J Sport Rehabil. 2019;28:266-271. doi:10.1123/jsr.2018-0131

10. Murphy M, Stockden M, Withers K, Breidahl W, Charlesworth J. Eight-week return to play following latarjet shoulder reconstruction in an Australian football player: a case report. J Sport Rehabil. 2019;28:283-287. doi:10.1123/jsr.2017-0194

11. Fairbairn JR, Huxel Bliven KC. Incidence of shoulder injury in elite wheelchair athletes differ between sports: a critically appraised topic. J Sport Rehabil. 2019;28:294-298. doi:10.1123/jsr.2017-0360

12. Melo FCM, de Lima KKF, Silveira APKF, et al. Physical training and upper-limb strength of people with paraplegia: a systematic review. J Sport Rehabil. 2019;28:288-293. doi:10.1123/jsr.2017-0062 